\title{
The dynamics of touch sensing studied in a mass-spring-damper model of the skin
}

Udaya Rongala ${ }^{1}$, Andre Seyfarth $^{2}$, Vincent Hayward ${ }^{3}$, Henrik Jörntell $^{1}$

1: Neural Basis of Sensorimotor Control, Department of Experimental Medical Science, Lund University, Lund, Sweden

2: Lauflabor Locomotion Laboratory, Institute of Sports Science, Technical University of Darmstadt, Darmstadt, Germany

3: Actronika SAS, and ISIR, Sorbonne Universite, 27063 Paris, Seine, FranceParis, France

\section{Acknowledgements}

This work was supported by the EU H2020 Grant FETOpen project\# 829186 , 'ph-coding' (Predictive Haptic COding Devices In Next Generation interfaces) 


\begin{abstract}
It has been shown that the skin can provide highly resolvable, dynamic tactile information to the central nervous system. However, currently available skin models do not provide a matching level of dynamic complexity. Motivated by recent observations that everyday interactions create a diversity of widespread travelling waves of multiple overlaid frequencies in the skin, we here model the skin as a 3D-distributed mass-spring-damper model. Shear forces across each spring were reported back as separate sources of information, on which we performed information content analysis using principal component analysis. We found that a wide range of settings of spring constants, dampening coefficients and baseline tension resulted in highly resolvable dynamic information even for simple skin-object interactions. Optimization showed that there were some settings that were more beneficial for a higher temporal resolution, i.e. where multiple independent interactions could be more easily resolved temporally. Whereas even a single sensor reporting a skin shear force with infinite precision by itself can achieve infinite resolution, biological sensors are noisy. We therefore also analyzed the resolution of force direction in the dynamic skin model, when their simulated signal-to-noise ratio was varied. We conclude that biological skin due to its inherent dynamics can afford a low spatial resolution of sensors (subsampling) while still maintaining a very high resolution for detecting skin-object interaction dynamics, and that biological evolution moreover due to this construct likely has been free to 'play' around with a variety of mechanical skin parameters and sensor densities without significantly compromising this resolution.
\end{abstract}




\section{INTRODUCTION}

The nature of dynamical skin-object interactions makes biological skin capable of rich, highdimensional sensing where each interaction is decomposable into a high number of dynamic, haptic input features [1]. These input features indeed appear to be represented within the central nervous system [2]. The key concept is that a rich diversity of relatively independent, temporal contact-state evolutions, i.e. input features, enables us to create high-dimensional, rich percepts of the outside world. Contact-state evolutions can be translated into spatiotemporal patterns of skin shear forces, which can be accessed by the brain if they are sampled and reported back to the central nervous system via the tactile skin sensors. The nature of such skin shear forces, and how they evolve over time, is to a large degree dependent on the internal dynamical properties of the skin. In order to better understand the richness of mechanical effects that can arise in biological skin, we wanted to create a skin model designed to capture central dynamic components of biological skin, without being too computationally costly.

The mass-spring-damper model is one approach to model mechanical dynamics that can be said to have many traits in common with the skin. In particular the observation that everyday interactions generate widespread travelling waves of multiple overlaid frequencies [3] speaks in favor of that the mass-spring model is a reasonable approximation of skin dynamics. The skin is composed of distributed masses, i.e. tissue, that are connected with dampened springs (connective tissue, [10]), and which can have a varying degree of tension, for example depending on joint angles or musclecontrolled pressure between skin and object. The mass-spring-damper model can be constructed in a grid-like arrangement with varying degrees of resolution, but is computationally less expensive than finite element models (FEMs) of the skin. Mass-spring-damper models are less detailed than FEMs, but we argued that they can still be providing sufficiently complex dynamics to capture the essential features of skin properties in skin-object interactions. 


\section{METHODS}

\section{Skin Structure}

The skin model was designed as a mass-spring-damper model, where the adjacent masses were connected using springs and dampers. The masses in this skin model were arranged in three layers, mimicking the structural properties of human skin (epidermis, dermis, and hypodermis). In each layer, the masses were homogeneously distributed in a mesh grid structure, and across the layers the masses were arranged in a pyramidal structure (Figure 1A). Such distribution of masses allowed the skin structure to have symmetrical geometries to implement the finite element method using stiffness matrix (see below). The skin model studied in this article has a mesh size of $6 \times 6$ masses in layer 1 (36 masses), $5 \times 5$ masses in layer 2 ( 25 masses) and 4x4 masses in layer 3 (16 masses). The masses on the edges of the skin model (in all layers) were fixed in all three axes. Additionally, all the masses in the third layer were fixed in all three axes.

\section{Mass-spring-damper model}

The equation of motion of a single degree of freedom mass-spring-damper system is given by,

$$
m \ddot{x}(t)+c \dot{x}(t)+k x(t)=F(t)
$$

Where $k$ is the stiffness of the spring, $m$ is the mass, $c$ is the dampening coefficient and $F$ is the driving force. $x, \dot{x}$ and $\ddot{x}$ are the position, velocity, and acceleration of the mass respectively. The equation 1 can be re-written for a multiple degree-of-freedom system, where the scalar variables are replaced with matrices and vectors,

$$
M \ddot{q}+C \dot{q}+K q=f
$$

Where $M, C$ and $K$ are symmetric matrices denoting mass, stiffness and damping of the overall system. $f$ is the input force vector. $q, \dot{q}$ and $\ddot{q}$ are the vectors indicating the position, velocity, and acceleration.

Furthermore, to compute the numerical integration (to perform mathematical simulations), the above equation of motion is written in state-space form as below [4],

$$
\dot{x}(t)=A x(t)+B u(t)
$$

Where $x$ is the state vector, $u$ is the applied input force, $A$ is the state matrix, and $B$ is the input matrix. Further coupling equation 2 to equation 3 , we achieve

$$
M \ddot{q}(t)=-C \dot{q}(t)-K q(t)+f(t)
$$

Which can be written as below [4],

The state matrix $A$ is,

$$
\ddot{q}(t)=\left[\begin{array}{cc}
0 & I \\
-M^{-1} K & -M^{-1} C
\end{array}\right] x(t)+\left[\begin{array}{c}
0 \\
M^{-1}
\end{array}\right] f(t)
$$


The input matrix $B$ is,

$$
A=\left[\begin{array}{cc}
0 & I \\
-M^{-1} K & -M^{-1} C
\end{array}\right]
$$

and

$$
B=\left[\begin{array}{c}
0 \\
M^{-1}
\end{array}\right]
$$

$$
x=\left[\begin{array}{l}
q \\
\dot{q}
\end{array}\right]
$$

To compute the numerical solution of the above-described motion equation, an ode45 solver was used using MATLAB. The ode45 solver uses a fifth order Runge-Kutta method. Each mass within the skin model can move in the three-dimensional space ( $x, y$ and $z$ axes), the matrices and vectors in above defined state equation were adapted accordingly.

\section{Stiffness matrix}

The stiffness matrix $(K)$ was constructed by grouping neighboring masses (connected with springs) into triangular configuration as in the finite element method (FEM). This grouping allows us to describe the structural relationship between all the springs within the skin. In each layer, a group of four neighboring masses were configured into two triangles using five springs as illustrated in Figure 1B (springs between M5-M6-M11 and M6-M11-M12). Across the layers, two neighboring masses in layer 1 form a triangular configuration with the mass beneath in layer 2 (along z-axis), as illustrated in Figure 1C (springs between M5-M6-M41 and M11-M12-M41) and Figure 1A (with dotted lines). An example of stiffness matrix for the mass-spring-damper configuration shown in Figure 1B, is available in the Supplementary Material. The stiffness matrix for the entire skin model is presented in Supplementary Material code.

\section{Force vector (Initial force)}

To simulate the skin model dynamics, we defined the input force vector $(f)$ of equation 5 as an exponential decay function (Figure 1D). The input force vector was three dimensional $(f=$ $\left[f_{x} f_{y} f_{z}\right]$ ), and was varied depending on the type of input stimulus (Figure $3 \mathrm{~A}-\mathrm{E}$ ) presented. We tested five different input stimuli (Figure $3 \mathrm{~A}-\mathrm{E}$ ) where each stimulus had different force conditions (direction of force indicated by red arrows in Figure 3) applied on specific masses in layer 1 . In Figure $1,2 \&$ S1, the results discussed were based on the input stimulus \#1, where the input force along the $z$ axis was applied to a single mass (M15) in layer 1 . The phase (time period) at which an active force was applied to the masses was defined as the indentation phase, and rest of the simulation time was defined as the post-indentation phase (Figure 1D).

\section{Sensory responses / Spring forces}

The forces across each spring were considered as the sensory output signals of the skin model. The force across each spring $\left(F_{S}\right)$ was given by Hooke's law $\left(F_{S}=k x\right)$. Where, $k$ is the stiffness constant of the spring and $x$ is the rate of change from its equilibrium position. For the configuration of the skin model presented in this article, we had a total of 338 springs resulting in 338 time-continuous sensory outputs. 


\section{Spiking neuron model}

The Izhikevich neuron model [5] was used to mimic mechanosensory like spiking responses (Figure 4B) for given sensory input (spring forces, Figure 4A). For Izhikevich neuron model, the membrane potential $(v)$ and the adaptation variable $(u)$ were updated via the following nonlinear differential equations discretized using Euler's method.

$$
\begin{gathered}
\dot{v}=A v^{2}+B v+C-u+(I * K) \\
\dot{u}=a(b v-u)
\end{gathered}
$$

When the membrane potential $(v)$ reached the spike depolarization threshold of $30 \mathrm{mV}$, one spike was produced followed by a reset:

$$
\text { if } v>30 m V \text {, then }\left\{\begin{array}{c}
v \leftarrow c \\
u \leftarrow u+d
\end{array}\right.
$$

The $A, B$, and $C$ parameters and the spiking threshold were the standard ones of the Izhikevich artificial neuron model, whereas the parameters $a, b, c$, and $d$ were selected (Table 1 ) to mimic a regular spiking behavior $[5,6]$. I was the input current to the neuron model, that was skin sensory responses (spring forces) in this article and $K$ is the input gain factor.

\section{Stochasticity in spiking neuron models}

Izhikevich neuron model is a deterministic system that generates stiff responses for the same input. This neuron model behavior is unlike the stochastic behavior of tactile afferent spike responses observed in humans [7]. Therefore, to achieve the stochastic neuron model properties, we have added a noise to the neuron model variables (membrane potential $(v)$ and resting potential $(u)$ ) [8]. This noise is generated as gaussian white noise, whose level can be adjusted by signal noise ratio $(\mathrm{SNR})$ in $\mathrm{dB}$. The stochasticity in the neuron model is given by,

$$
\begin{aligned}
& v=A W G N(v, S N R) \\
& u=A W G N(u, S N R)
\end{aligned}
$$

We used the inbuilt MATLAB function "awgn" to generate this gaussian white noise. It adds this noise with the relative signal-to-noise ratio to a signal.

\section{Spike convolution}

The Izhikevich neuron model spike output was convoluted to resemble post-synaptic potential (time continuous activity) using the following kernel equation,

$$
a=\frac{\tau_{m}}{\tau_{d}-\tau_{r}} *\left[\exp \left(\frac{-t-\tau_{l}-t^{*}}{\tau_{d}}\right)-\exp \left(\frac{-t-\tau_{l}-t^{*}}{\tau_{r}}\right)\right]
$$

Where, $t^{*}$ is the input spike time, $\tau_{d}$ is the decay time (4 ms), $\tau_{r}$ is the rise time (12.5 ms) and $\tau_{m}$ is the constant to calculate ration between rise time and decay time $(21.3 \mathrm{~ms})$, and $\tau_{l}$ is the latency time which is zero in this case. These values were chosen based on the previous work [9].

Table 1: Izhikevich neuron model parameters used in the evaluation of this study.

\begin{tabular}{|l|l|l|l|l|l|l|l|}
\hline A & B & C & a & b & c & d & K \\
\hline 0.04 & 5 & 140 & 0.02 & 0.2 & -65 & 9 & 500 \\
\hline
\end{tabular}




\section{Analysis methods}

\section{Principal component analysis}

The principal component analysis (PCA) was used to determine the amount of unique information present in the sensory responses during different sensing phases (indentation / post-indentation) for a given skin property. We used the inbuilt MATLAB function "pca" to perform this analysis. In brief, the pca function projects the input data onto principal component space (new set of variables) that explain the high dimensional input data on fewer output dimensions that encompass the maximum preservation of information in the input data. The "pca" function additionally returns the "explained variance", which express the percentage of information each principal component explains.

In this article, we performed PCA analysis on all the sensory responses for indentation and postindentation phase. We utilized the variance explained for each principal component to assess the amount of information present in the sensory responses during indentation /post-indentation phases (Figure 1F, S1). The higher the number of principal components needed to explain a given level of variance in the information ( $99 \%$ for example), the richer the information content in the sensory responses.

\section{Area computation}

Supplementary figure 2 illustrates the definition of the area between the information curves for the indentation and the post-indentation phases, respectively, across the principal components (PCs). This analysis provided data on the discrepancy in information content in first $P C(s)$ during the indentation and the post-indentation phases, respectively. If the area value was negative (Figure $2 \mathrm{~A}$ ) then the indentation phase had a lower variance explained by the first PC(s) compared to the postindentation phase. This would in turn imply that a higher number of $\mathrm{PC}(\mathrm{s})$ was required to explain the total variance in the indentation phase, which in turn would indicate that there was more information in the indentation phase than in the post-indentation phase.

\section{$P C$ ratio}

The number of PCs (Figure 2B) needed to explain $99 \%$ of the variance in the information, expressed as the ratio between the indentation and the post-indentation phases (equation 9), was used as another measure of the richness of information in the indentation phase. A high value of this "PC ratio" indicated that a higher number of PCs were needed to explain the information during the indentation phase compared to the post-indentation phase.

$$
\text { PC ratio }=\left(\sum P C\right)_{\text {Indentation }} /\left(\sum P C\right)_{\text {Post-indentaiotn }}
$$

\section{Signal information ratio}

The signal information for first 3 PCs, expressed as the ratio between the indentation and the postindentation phases (equation 10), was used as another measure of the richness of information in the indentation phase. A high value of this "Signal information ratio" indicated that a higher variance was needed to explain the information during the indentation phase compared to the post-indentation phase. 
Signal information ratio

$=\mu($ variance $\%) / \sigma(\text { variance } \%)_{\text {Indentation }} / \mu($ variance $\%) / \sigma(\text { variance } \%)_{\text {Post-indentaiotn }}$

\section{Cross correlation measure}

The correlation index measure was used to compute the similarity of the sensory responses (Figures 3F). The correlation between two signals was computed with an inbuilt MATLAB function "xcorr" (with zero lag), which produces values from 0 (uncorrelated) to 1 (identical).

\section{Classification algorithm}

For classification we chose a linear support vector machine (SVM) method trained with supervised learning. The classifier was trained and tested using a 5-fold cross-validation, which was repeated for 100 iterations to ensure the robustness of the classifier and training procedure. We used the inbuilt MATLAB toolbox "Classification Learner" to perform this computation. The convoluted timecontinuous responses of Izhikevich neuron model output (for 50 repetitions across 9 different indentation conditions, Figure 4D-F) were chosen as input to the above-mentioned classification algorithm. The input vector data was binned into 9 classes, representing all the 9 different indentation force conditions to be identified by the SVM classifier. 
bioRxiv preprint doi: https://doi.org/10.1101/2021.09.06.459180; this version posted September 6, 2021. The copyright holder for this preprint (which was not certified by peer review) is the author/funder. All rights reserved. No reuse allowed without permission.

\section{Results}

\section{Outline of the skin model}

Figure 1 illustrates the general properties of the skin model. Nodes are representing the masses. All masses are connected to their nearest neighboring masses with springs and dampeners, which would correspond to the mechanical effects of skin connective tissue [10]. Gravity was not simulated, as we argued that the intrinsic tension of the skin must be greater than the impact of gravity on the skin masses - otherwise, the skin would report differently on the same interaction depending on the orientation of the skin. The skin model was composed of three layers (which can be thought of as the epidermis, dermis, and hypodermis), where the deepest layer consisted of fixed nodes (a simplification of the connective tissue underlying the skin). The lateral edges of the simulated skin were also composed of fixed nodes. Nodes within a layer were connected in triangles (Figure 1B), and also the connections between nodes across adjacent layers were triangular hence forming pyramids of interconnections (Figure 1C). Skin object interactions were simulated by simply pushing or pulling on a subset of the masses in the superficial layer. This is a simplification compared to reality, where the sensors are not immediately accessible from the surface of the skin, so the only skin components immediately affected by external forces are the outermost layer of the epidermis. The frictional forces that arise in the skin-object interactions in this surface layer cause the skin tissue displacement and the skin shear forces internally in the skin where the sensors are located. In our experimental setting, stimulations consisted of abrupt initial force followed by a gradual tapering of that force (Figure 1D).

A

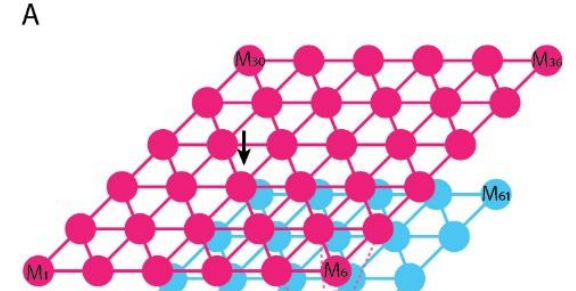

B
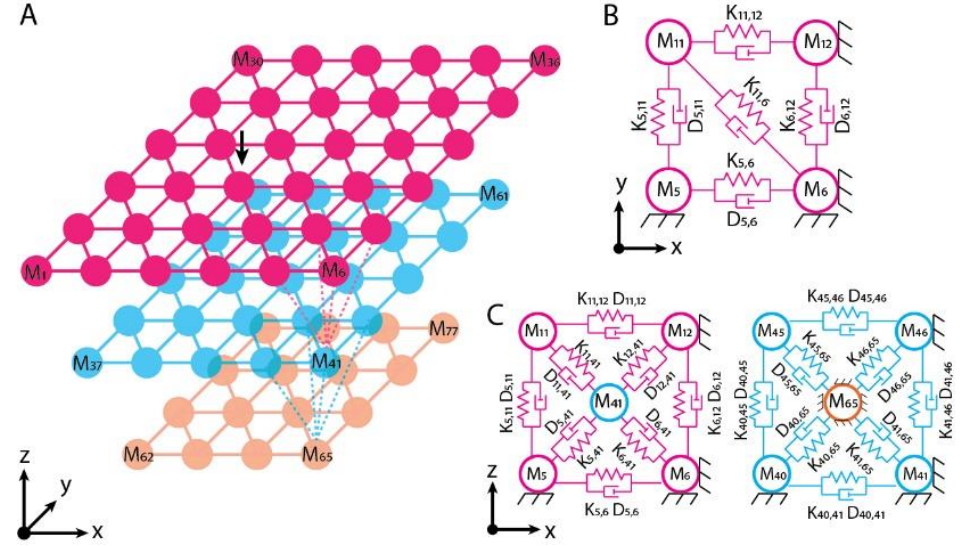
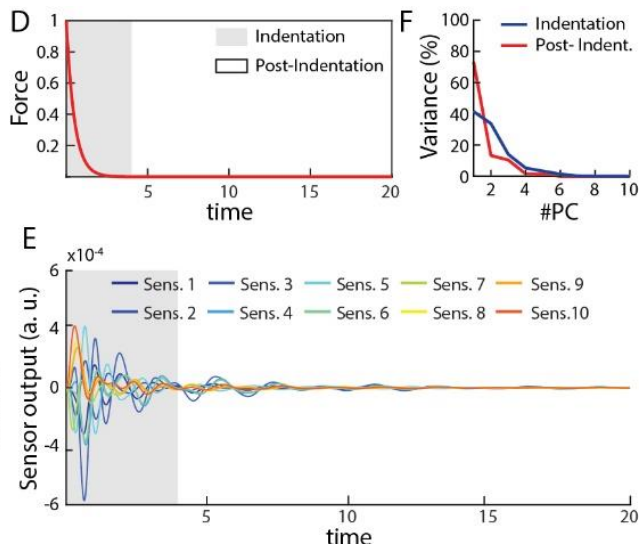

Figure 1. Skin model and example sensor responses. (A) Schematic of the three-layer skin model. The skin model was designed as a mass-spring-damper model, where the masses within each layer were homogeneously distributed in a mesh grid structure and interconnected by springs and dampers. Across layers (along the z-axis), the masses were arranged in a pyramidal structure (layer 1: 36 masses (6x6 mesh), layer 2: 25 masses (5x5 mesh) and layer 3: 16 masses (4x4 mesh)). The masses between layers were also interconnected using springs and dampers (as illustrated with dotted lines). The masses on the edge of the skin (in all layers) were fixed in all three axes. Additionally, all the masses in the third layer were fixed in all three axes. (B) The spring-damper arrangement between masses within the same layer. In this and all subsequent figures, each spring was also a sensor and the force across the spring was the sensor signal. (C) The spring-damper arrangement between the masses across different layers. (D) The force profile used to displace the given mass (M15, see arrow in $\boldsymbol{A})$ to elicit the skin dynamic effects. The set of different spatial force patterns used later in this study (Fig. 3) were all provided with this time profile. The shaded zone indicates the time range in which the forces (arbitrary units) were applied to the skin sensors (Indentation Phase). (E) The sensor responses (spring forces, see Methods) for an indentation (with the force profile given in $\boldsymbol{D}$ ) applied to a single mass in layer 1 (M15, indicated with a black arrow in $\boldsymbol{A})$. In this illustration, we show only the responses of sensors that were connected to Mass \#15. (F) PCA analysis across all of the 338 sensors shows the amount of information present across the skin sensor population during the indentation phase (grey zone) and the post-indentation phase (white zone). These curves show the amount of variance in the sensory information that was explained by each principal component. For example, six PCS were needed to explain 99\% of sensory information during indentation phase, compared to four PCs that were needed to explain $99 \%$ information post-indentation phase. 

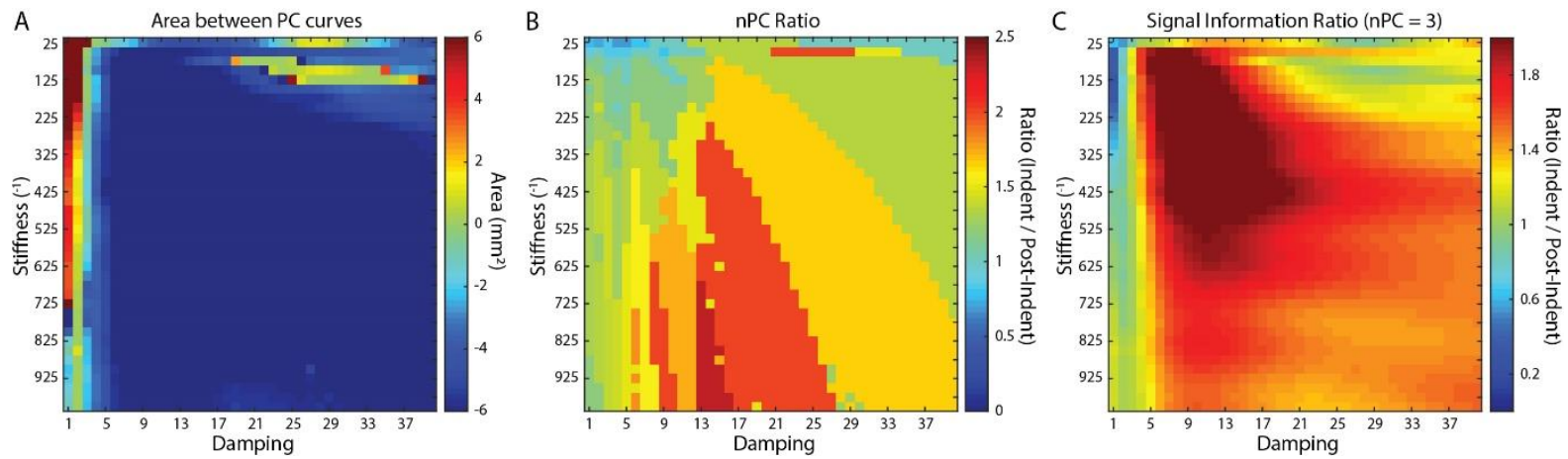

Figure 2. Optimization of skin dynamics parameters. These plots illustrate the effects of mechanical properties of the skin (spring stiffness (K) and dampening coefficient (D)) on the sensory information as evaluated by the PCA (Fig. 1E) of skin sensor information during the indentation and the post-indentation phases. (A) Computed area between the PC curves (for indentation and post-indentation phases) as illustrated in Suppl. Figure 2. The sensory responses were generated by input stimulus \#1, where input force was applied to a single mass (M15) along the z-axis in layer 1 (as in Figure 1). (B) Ratio of the number of PCs needed to explain 99\% of information between indentation and post-indentation phase, where a high value indicates a proportionally lower information content in the post-indentation phase. (C) Ratio of the signal information (see Methods) for the first 3 PCs between indentation and the post-indentation phase.

Since skin sensors sense shear forces, we used the load across each spring as the unitary tactile sensor data. On stimulation, due to the intrinsic dynamics of the skin model, the spring forces were strongest during the stimulation but also remained present long after the termination of the force application (Figure 1E). Moreover, each sensor reported dynamic information that was to some extent unique (Figure 1E; only a subset of the nearest neighboring sensors is shown). In order to analyze the information content, we used principal component analysis of the activity distribution across the entire population of sensors (Figure 1F). The PCA was performed across all sensing conditions for fixed time windows, so that we could track the variance explained by each PC across each unit of time and thereby compare the depth of the PCs engaged across conditions and time. In general, if a higher number of PCs were required to explain the variance of a sensor population activity for a given time window, this was taken as an indication that the time window contained a richer information.

\section{Parameter optimization}

The mass-spring-damper model inevitably results in that there will be dynamic after-effects after cessation of an active skin-object interaction (i.e. with resulting forces). Such dynamic after-effects are potentially containing information about the nature of the interaction that just took place, but will gradually be superseded by main features of the skin dynamics that will be common to the aftereffects of all types of interactions, hence containing less specific information. To avoid mixing up interaction-specific dynamic features with pervasive intrinsic skin dynamic features, and to increase the temporal resolution of reporting sequentially different types of interactions, we first argued that for the skin, nature must have found a trade-off between richness of information and temporal resolution. Therefore, we did a parameter optimization to find out the combination of spring constants, dampening coefficients and skin tension that provided the most information during active contact in relation to the information present in the after-effects of such active interactions (blue and red curves, respectively, in Fig. 1F).

As a rule, during active indentation the sensor population generated variance across a higher number of PCs than in the post-indentation phase. The post-indentation phase instead provided its information preferably in the lower order PCs, i.e. it contained more of the main features/responsive modes of the skin. We first used this discrepancy to quantify the higher richness of information in the 
active indentation, by simply measuring the area between the curves for the two phases (Suppl Fig 2 ). In Figure $2 \mathrm{~A}$ it is shown that for most of the parameter settings for dampening and spring constants, this area did not vary greatly except when the dampening was extremely low or the spring was highly stiff. As another measure, we simply used the number of PCs (nPC) required to explain $99 \%$ of the variance in the indentation phases and in the post-indentation phase, respectively, to obtain a nPC-ratio between the two (Figure 2B). In this case, we found that a high nPC-ratio was obtained for intermediate dampening and high spring compliance. In contrast, for the ratio of signal information (Figure 2C) we found a low spring compliance to be beneficial, while intermediate

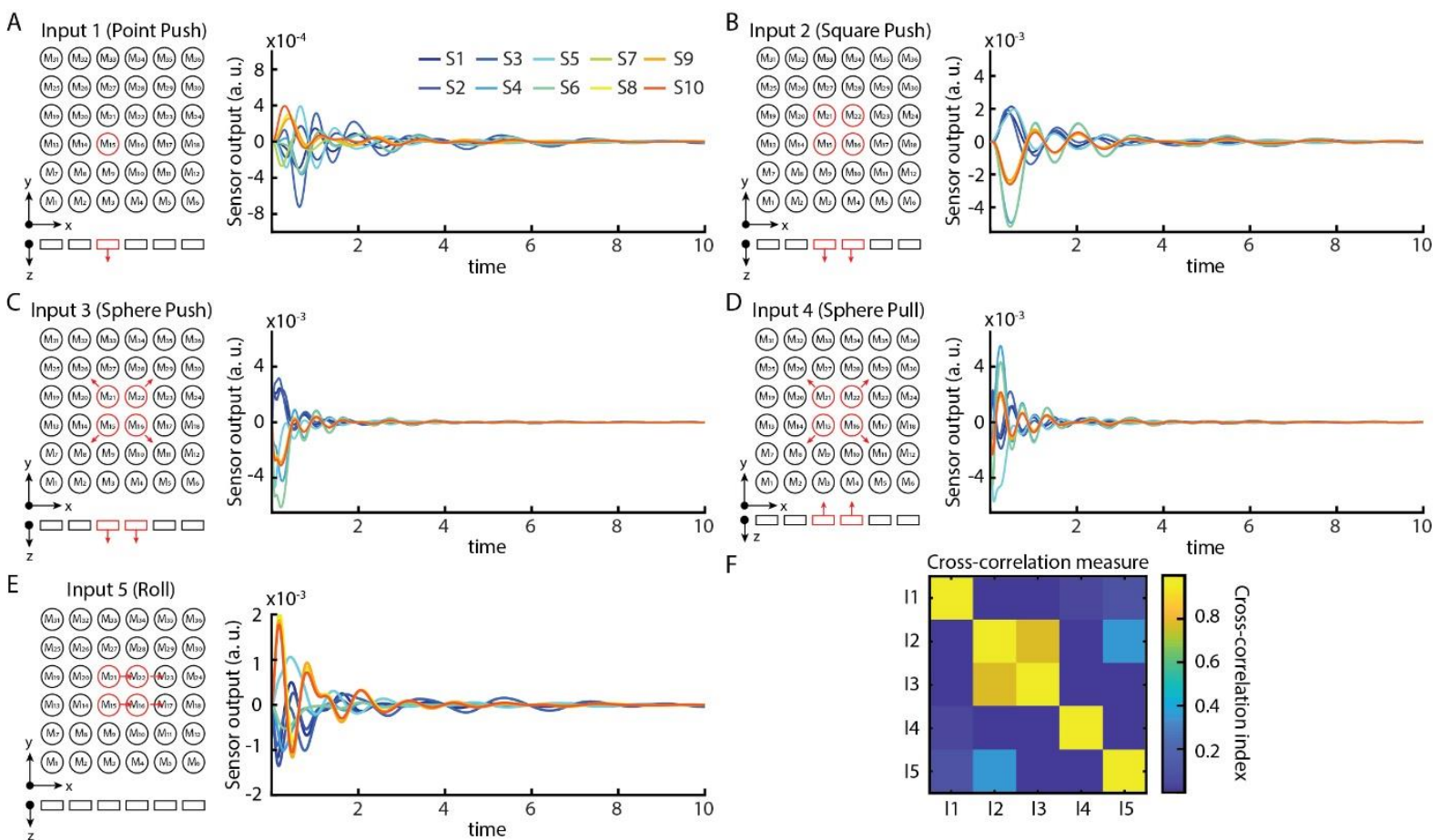

Figure 3 Sensory responses for different stimuli. (A - E) Five different input stimuli (as indicated in the sketches on left side of each plot) were tested, each having different initial force conditions (direction of force indicated with red arrows) applied on specific masses in layer 1 (red circles). The right side of each plot shows the sensor responses for the given input stimulus. In this illustration, we show only the responses of sensors that were connected to mass M\#15. (F) Confusion matrix of the cross-correlation measure for the output of each single sensor across the five different input stimulus conditions. The values reported here are the mean values across all the sensors. Cross-correlations of 1 signify a high similarity between the signals of individual sensors across the different stimulus conditions whereas 0 signifies high dissimilarity.

dampening was beneficial also in this scape.

Overall, this analysis showed that there is a very broad landscape of useful solutions, where the skin dynamics of the mass-spring-damper model can provide rich information. For the remainder of the paper we picked one specific parameter combination (intermediate dampening, high-intermediate stiffness) which provided relatively high temporal resolution (Figure $2 \mathrm{C}$ ) but which was also rich in information for the indentation phase (Figure 2B). We first tested different input stimuli which were created by varying the direction of the force vector(s) applied to one mass or a group of masses within layer 1, while keeping the same temporal profile (Figure 1D) (Figure 3A-E). The sensor data, represented by a subset of 10 sensors being in direct connection with the moved mass (M\#15), was clearly different across these five conditions. Figure $3 \mathrm{~F}$ shows this quantitatively by a crosscorrelation measure across these 10 sensors. Notably, this analysis indicated a higher degree of 
similarity between the sensor responses to Inputs 2 and 3 than between the rest, which can be explained by that these two inputs were more similar in terms of the forces applied, the only difference being that for input 2 the direction of the 'pushing' force had an angle against the $z$ axis. Note that due to the fact that the model had dynamics, there were still forces arising in the $x$-y plane also in the case of the perpendicular force applied in input 2.

\section{High-precision decoding depending on the temporal resolution of the skin sensors}

Decoding depends on the reliability of the skin sensor output. If each skin sensor has no noise (as in a deterministic system), and has an infinite precision, then one sensor would be enough to achieve infinite resolution for sensing. This will be the case even for a static system; imagine for example a single node attached to a spring with a fixed base. If a force is applied to extend the spring along the $x$ axis, and then next the same force, but now with a minimal angle deviation from the $x$ axis, is applied then the deviation will mean that the force vector along the $x$ axis has now become smaller. Hence, if the spring has infinite resolution, i.e. no matter how small the force difference is, the sensor will be able to report that small difference. Then the sensor has infinite resolution for the force angle. If the absolute level of force is not known, then disambiguation of force direction could be solved by a larger number of springs, with different orientation. In a system where forces are distributed across a large number of springs, those same effects would be in effect and it could in principle be enough with two sensors to sense any force direction and force angle. In a dynamical system with a large number of dynamical springs, the same effects plus the system dynamics would be expected to be in effect, where the complication for the static sensing task would be that the signal required to detect the force angle may be overlaid with internal dynamic modes of the skin. 
bioRxiv preprint doi: https://doi.org/10.1101/2021.09.06.459180; this version posted September 6, 2021. The copyright holder for this preprint (which was not certified by peer review) is the author/funder. All rights reserved. No reuse allowed without permission.

This is shown in Figure 4, where Figure 4A shows the responses of 10 sensors to one input. Figure 4B illustrates that even one of these sensors (\#5 in this case) can be used to tell a variety of stimuli apart. Again, if the sensor has infinite precision, it will have infinite resolution so no matter how small the differences in force vector directions, this one sensor would be enough to differentiate them. However, biological sensors have neural properties, i.e. they mediate their sensor signals with spike output. In neurons, the average intervals of the spikes approximately linearly reflect the amount of excitatory signal across the membrane [11], but each individual interval is subject to a certain level of stochasticity [12]. Hence, due to the inherent noise in neural spike signals, the spike output patterns are approximations of the continuous underlying receptor potential (receptor potentials for the 10 sensors illustrated are given in Figure 4A). The temporal precision with which the skin sensor can reliably report information then becomes the limit of resolution of the sensing. This is shown for the 10 sensors in Figure $4 C$, where an example of the spike responses across the 10 sensors are illustrated and for the example sensor (\#5) across 50 repetitions in Figure 4D. In this case, the
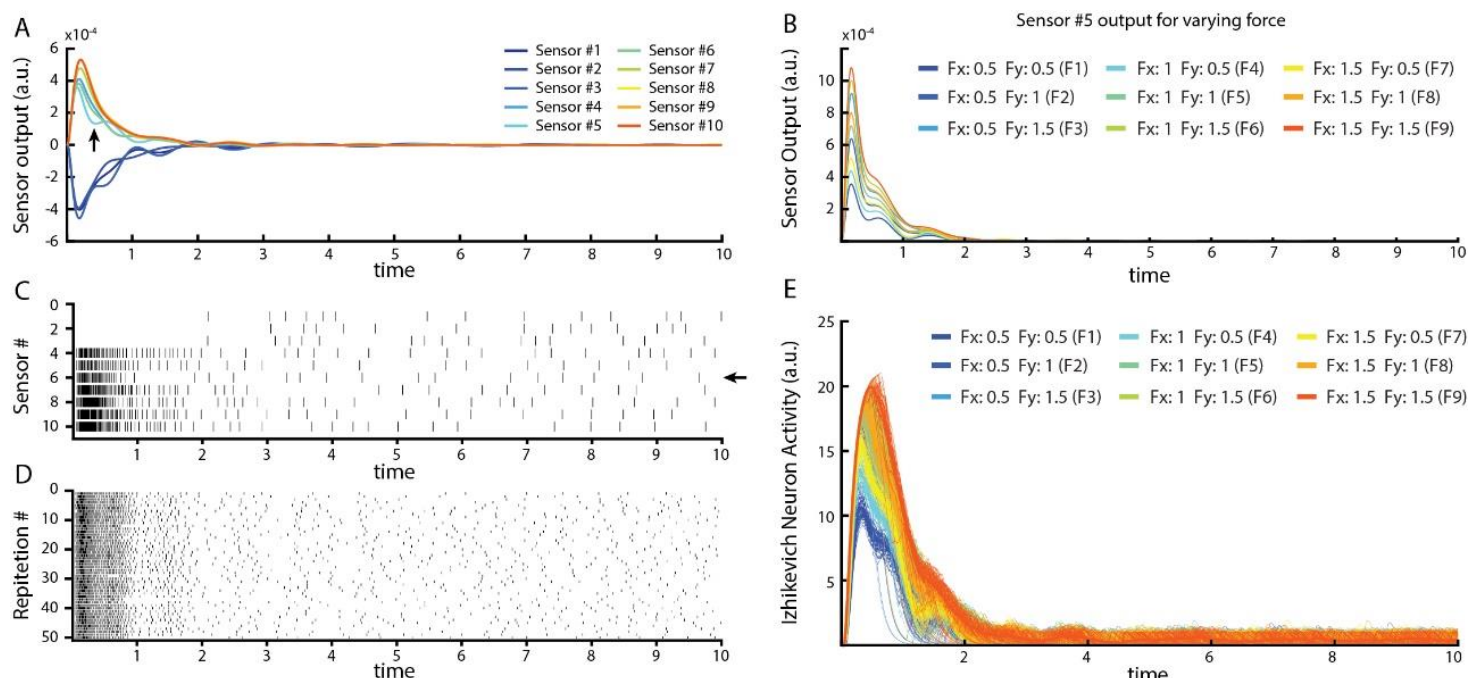

$\mathrm{F}$

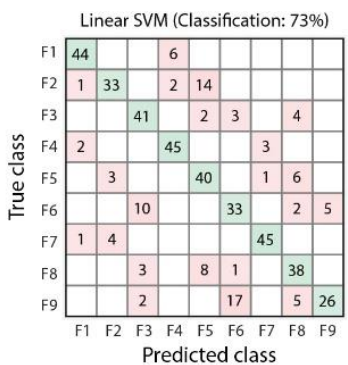

G

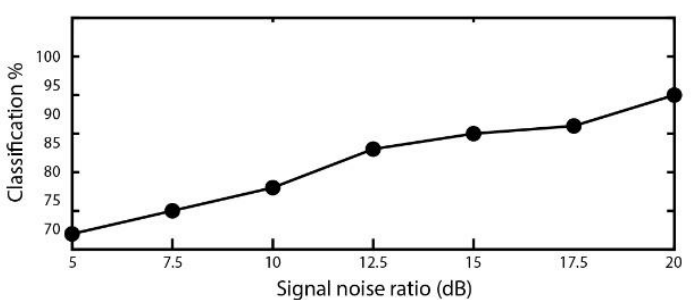

Figure 4. Differentiability of sensor signals and complications caused by spiking. (A) The sensor responses for an indentation (with the force profile given in Figure 1D) applied to a single mass along the $x$-axis $(F x=0.5 N)$ and $y$ axis $(F y=0.5 \mathrm{~N})$ in layer $1(\mathrm{M} 15)$. In this illustration, we show only the responses of the 10 sensors that were connected to Mass \#15. (B) For sensor \#5 receptor potential (spring force) without spiking, the responses to indentations with varying forces and angles along the $x$ and $y$ axes. (C) The spike output of a stochastic Izhikevich neuron model driven by the sensory signals in $\boldsymbol{A}$, one spike train for each sensor. (D) Izhikevich neuron model output for 50 repetitions of the exact same signal (from sensor \#5 in A). This raster plot shows the stochastic firing behavior of the Izhikevich neuron model for a signal-to-noise ratio of $10 \mathrm{~dB}$ (see Methods). (E) For the same forces and angles as in B, spiking outputs from sensor \#5 generated by the Izhikevich neuron model output for 50 repetitions with a signal-to-noise ratio of $10 \mathrm{~dB}$. The spike responses were convoluted using a kernel function (see Methods). (F) A confusion matrix showing the classification performance for the Izhikevich neuron responses for 50 repetitions (the same responses as in E) under the 9 different indentation conditions. The classification was performed using Linear Support Vector Machine (SVM), with 5-fold validation. (G) The classification performance of the Izhikevich neuron responses across 9 different indentation conditions. Here we test the classification performance (the average in the diagonal of $\boldsymbol{F}$ ) for the spiking neuron model at different settings for the signal-tonoise ratio. 
differentiation of the force angles becomes more difficult, because the integrated signal of those spike responses will differ between repetitions and so differentiation becomes less reliable (Figure $4 \mathrm{E}$ ). The reliability of signal differentiation was evaluated by an SVM classifier to give a quantitative example (Figure $4 \mathrm{~F}$ ). The classification was well below 100\%, even though the angular differences between the stimuli were relatively large. This resolution would in turn vary with the nature of the stimulus, where we here used a very brief force peak with a gradual decay. Finally, a higher resolution was naturally achievable with a better signal-to-noise ratio in the spike encoding in the sensor (Figure 4G). 


\section{DISCUSSION}

Here we introduced a dynamical skin model to better understand the potential effects that can arise in biological skin during haptics interactions and what advantages those effects could have for information richness. This work builds on that the biological skin is a dynamic entity and that the biomechanics of skin plays an important role in defining the relation between tactile interactions and mechanosensory responses. The mechanical properties of biological skin vary greatly regionally within the same individual [13], between individuals (i.e. for example related to age; [14]) and even more across species. In our model, these mechanical properties were to a large extent defined by the parameter settings of the spring constants $(K)$ and the damper coefficients $(C)$. It is possible that actual skin could have been more precisely captured with more extensively fitted spring constants and damper coefficients adapted for a specific type of skin. However, our aim was primarily to illustrate the implications of the concept of the skin as a dynamical system, and our initial analysis (Figure 2) showed that useful effects arose across a broad spectrum of parameter settings.

The main advantage of a dynamical skin is that the information generated from it is richly resolvable, more so than in a static system. Hence, it merely requires that sensors have a distribution within this tissue, and the brain can potentially learn to perceive/separate a variety of mechanical effects. Since this effects are separable into relatively independent components, as indicated by PCA (Figures 1 and 2 ), these effects could also potentially be combined thus enabling extremely rich perceptual capability. Notably, in normal behavior, skin-object interactions typically are highly dynamic and last for $100 \mathrm{~s}$ of $\mathrm{ms}$, which hence can result in multiple overlaid dynamical effects that are specific to the skin-object interaction (Figure 3), which will be superimposed on the more generic internal harmonic modes of the skin. Full perceptual capability requires that the brain learns these internal harmonic modes, which hence will be part of the understanding of 'self', against which the specific superimposed modes generated in the interaction can be compared.

Even though a sensor with infinite precision can only exist in software, as we described in this paper such sensors, if they existed in reality and were located in a dynamical skin such as in our model, would be capable of carrying very rich information. In biology, however, sensors transfer information to the brain via spikes. Spikes require the neural process of spike generation, which is an extra filter imposed on the information transfer, and which does not have infinite precision (Figure 4). This is hence the main limiting factor for the information richness that the brain can access. However, due the inherent richness of the skin dynamics, the brain could still receive rich information, if the number of sensors distributed in the tissue becomes sufficiently high. Note that these issues apply in particular to situations with dynamic skin-object interactions, where sensors may spend a large part of the time close to their firing threshold and thus exhibiting a larger level of stochasticity $[11,12]$.

Several earlier skin models had the explicit aim to fit their models to recreate the skin afferent neural responses observed in experiments for given, reduced, input conditions. One of these models was a 3D finite element analysis (FEM) based model [15]. Here, a multi-layer skin model was constructed using a finite element mesh that was generated based on the macroscopic anatomical structures of both monkey and human fingertips. Another approach has been to use continuum mechanics $[16,17]$ to characterize the deformations in the tissue, which portrait stress as a quasistatic elastic model of the skin [18]. In contrast, here we used the mass-spring-damper model to achieve a computationally efficient skin model, designed according to the microscopic structural elements of the skin and to 
capture the rich skin dynamics that could occur under natural skin-object interactions, rather than fitting the model to recording data of skin afferent responses evoked by an arbitrary input.

Extensive research has also focused on creating bio-inspired electronic skin sensors for various haptic applications [19-22] and in neuro-prosthesis to establish tactile sensory feedback [23-26]. For example for skin-neuro-prostheses, which would be designed along the lines described by our skin model, could enable a richer sensation and hence elevating the usability of such prostheses.

\section{REFERENCES}

1. Hayward V. Is there a 'plenhaptic'function? Philos Trans R Soc B Biol Sci. 2011;366: 31153122.

2. Jörntell H, Bengtsson F, Geborek P, Spanne A, Terekhov A V., Hayward V. Segregation of tactile input features in neurons of the cuneate nucleus. Neuron. 2014;83: 1444-1452. doi:10.1016/j.neuron.2014.07.038

3. Shao Y, Hayward V, Visell Y. Spatial patterns of cutaneous vibration during whole-hand haptic interactions. Proc Natl Acad Sci U S A. 2016; 201520866. doi:10.1073/pnas.1520866113

4. Inman DJ. Vibration with control. John Wiley \& Sons; 2017.

5. Izhikevich EM. Simple model of spiking neurons. IEEE Trans Neural Networks. 2003;14: 15691572. doi:10.1109/TNN.2003.820440

6. Izhikevich EM. Which model to use for cortical spiking neurons? IEEE Trans Neural Networks. 2004;15: 1063-1070. doi:10.1109/TNN.2004.832719

7. Johansson RS, Flanagan JR. Coding and use of tactile signals from the fingertips in object manipulation tasks. Nat Rev Neurosci. 2009;10: 345-59. doi:10.1038/nrn2621

8. Yang Z, Gandhi V, Karamanoglu M, Graham B. Characterising information correlation in a stochastic Izhikevich neuron. 2015 International Joint Conference on Neural Networks (IJCNN). IEEE; 2015. pp. 1-5.

9. Rongala UB, Spanne A, Mazzoni A, Bengtsson F, Oddo CM, Jörntell H. Intracellular dynamics in cuneate nucleus neurons support self-stabilizing learning of generalizable tactile representations. Front Cell Neurosci. 2018. doi:10.3389/fncel.2018.00210

10. Woessner AE, Jones JD, Witt NJ, Sander EA, Quinn KP. Three-Dimensional Quantification of Collagen Microstructure During Tensile Mechanical Loading of Skin. Front Bioeng Biotechnol. 2021;9: 153.

11. Spanne A, Geborek P, Bengtsson F, Jörntell H. Spike generation estimated from stationary spike trains in a variety of neurons In vivo. Front Cell Neurosci. 2014. doi:10.3389/fncel.2014.00199

12. Nilsson MNP, Jörntell H. Channel current fluctuations conclusively explain neuronal encoding of internal potential into spike trains. Phys Rev E. 2021;103: 22407.

13. Rosicka K, Hill M, Wdowski MM. Skin anisotropy: Finding the optimal incision line for volar forearm in males and females. J Mech Behav Biomed Mater. 2021; 104805. 
14. Wilkinson HN, Hardman MJ. A Role for Estrogen in Skin Ageing and Dermal Biomechanics. Mech Ageing Dev. 2021; 111513.

15. Dandekar K, Raju BI, Srinivasan MA. 3-D finite-element models of human and monkey fingertips to investigate the mechanics of tactile sense. J Biomech Eng. 2003;125: 682-691.

16. Phillips JR, Johnson KO. Tactile spatial resolution. III. A continuum mechanics model of skin predicting mechanoreceptor responses to bars, edges, and gratings. J Neurophysiol. 1981;46: 1204-1225.

17. Sripati AP, Bensmaia SJ, Johnson KO. A continuum mechanical model of mechanoreceptive afferent responses to indented spatial patterns. J Neurophysiol. 2006;95: 3852-3864.

18. Saal HP, Delhaye BP, Rayhaun BC, Bensmaia SJ. Simulating tactile signals from the whole hand with millisecond precision. Proc Natl Acad Sci. 2017;114: E5693-E5702.

19. Dahiya R, Yogeswaran N, Liu F, Manjakkal L, Burdet E, Hayward V, et al. Large-area soft e-skin: The challenges beyond sensor designs. Proc IEEE. 2019;107: 2016-2033.

20. Kim D-H, Lu N, Ma R, Kim Y-S, Kim R-H, Wang S, et al. Epidermal electronics. Science (80- ). 2011;333: 838-843.

21. Fan JA, Yeo $\mathrm{W}-\mathrm{H}$, Su $\mathrm{Y}$, Hattori $\mathrm{Y}$, Lee $\mathrm{W}$, Jung $\mathrm{S}-\mathrm{Y}$, et al. Fractal design concepts for stretchable electronics. Nat Commun. 2014;5: 1-8.

22. Russell R. Compliant-skin tactile sensor. Proceedings 1987 IEEE International Conference on Robotics and Automation. IEEE; 1987. pp. 1645-1648.

23. Ouyang $Q$, Wu J, Shao Z, Chen D, Bisley JW. A Simplified Model for Simulating Population Responses of Tactile Afferents and Receptors in the Skin. IEEE Trans Biomed Eng. 2020;68: 556-567.

24. Wu Y, Liu Y, Zhou Y, Man Q, Hu C, Asghar W, et al. A skin-inspired tactile sensor for smart prosthetics. Sci Robot. 2018;3.

25. Osborn LE, Dragomir A, Betthauser JL, Hunt CL, Nguyen HH, Kaliki RR, et al. Prosthesis with neuromorphic multilayered e-dermis perceives touch and pain. Sci Robot. 2018;3.

26. Oddo CM, Raspopovic S, Artoni F, Mazzoni A, Spigler G, Petrini F, et al. Intraneural stimulation elicits discrimination of textural features by artificial fingertip in intact and amputee humans. Elife. 2016;5: e09148. 


\section{Supplementary Figures}

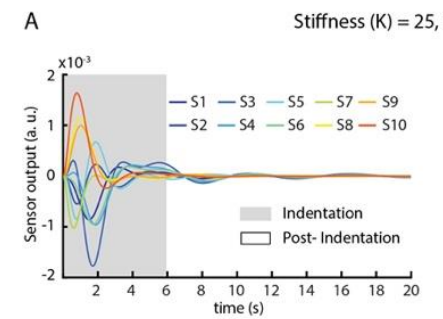

B

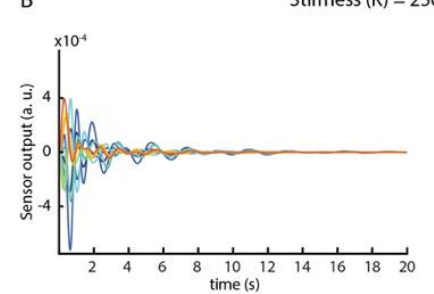

C

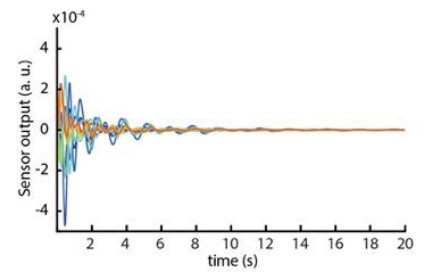

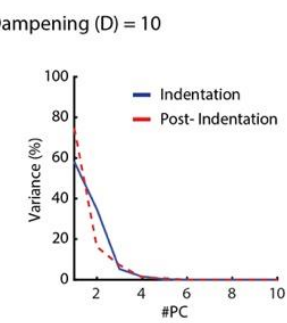

Dampening $(D)=10$

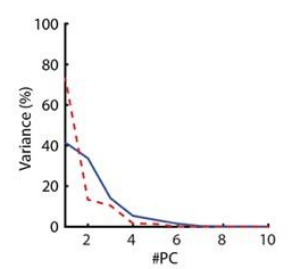

Stiffness $(K)=500$, Dampening $(D)=10$

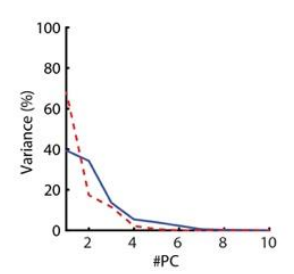

Input $=1$

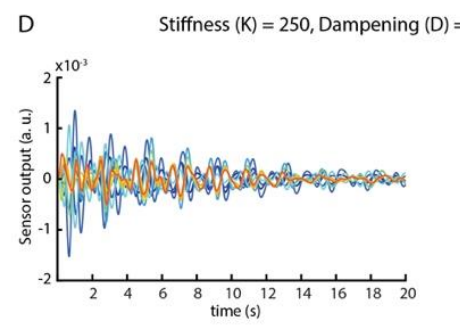

E

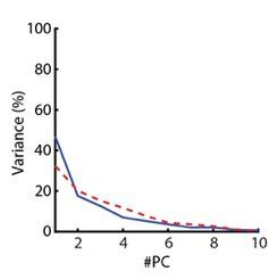

Stiffness $(K)=250$, Dampening $(D)=10$
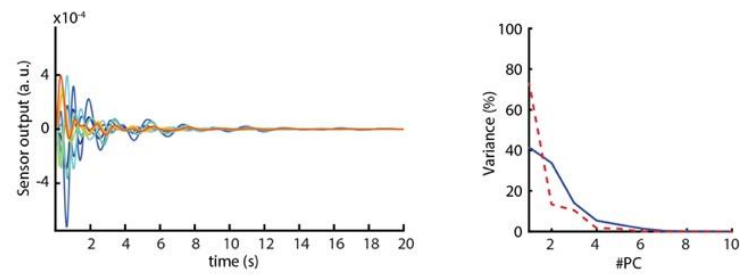

$\mathrm{F}$

Stiffness $(K)=250$, Dampening $(D)=20$

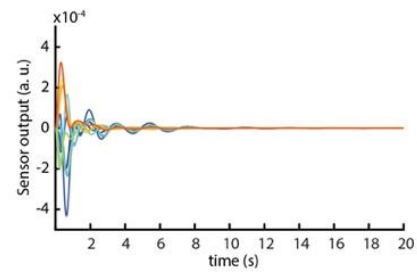

Supplementary Figure 1. Sensory information for various spring and damper coefficients. Left side of each plot illustrate the sensor responses for input stimulus 1. In this illustration, we show only the responses of sensors that were connected to Mass \#15. Right side of each plot show the PCA analysis across all the 338 sensor output responses. These curves indicate the amount of information present in the skin sensors (see Methods) during the indentation phase and post-indentation phase.

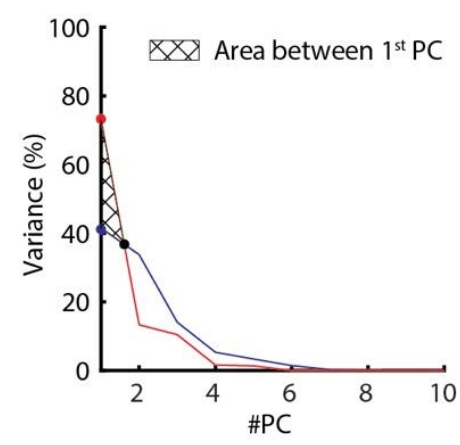

Supplementary Figure 2. Area computation between the information curves (for indentation and post-indentation phases) explaining variance for given PC 1. 